\title{
LA COMMUNAUTÉ DEPLACÉE. ÉCRITURE ET COMMUNAUTÉ DANS LE DIALOGUE ENTRE MAURICE BLANCHOT ET JEAN-LUC NANCY
}

\author{
Juan José Martínez Olguín \\ Universidad de Buenos Aires \\ Université Paris VIII \\ bttp://dx.doi.org/10.15304/ag.38.2.5610
}

\section{Resumen}

Con sus obras La comunidad desobrada y La comunidad inconfesable, Jean-Luc Nancy y Maurice Blanchot entablaron, en la década del 80, un diálogo cuyo objeto central fue el tema de la comunidad. En ese diálogo, sin embargo, no sólo estaban involucrados Nancy y Blanchot: ambos se reconocían como herederos de una reflexión que habían iniciado, con diferentes matices y en diferentes épocas, Nietzsche y Bataille. Varias décadas más tarde, en La comunidad revocada, Nancy vuelve sobre el debate que lo tuvo como protagonista en los ochenta: ¿qué tan lejos pretendió ir Blanchot con los argumentos que opuso contra el texto del propio Nancy? A partir de esa pregunta, que inicia uno de los apartados del último ensayo de Nancy, el trabajo se propone reanudar ese debate con el objeto de repensar el vínculo entre escritura y comunidad.

Palabras clave: comunidad, escritura, Blanchot, Nancy.

\begin{abstract}
With their texts The Inoperative Community and The Unavowable Community, JeanLuc Nancy and Maurice Blanchot started in the 80s a dialogue whose central object was the theme of the community. In this dialogue, however, not only Nancy and Blanchot were involved: both of them recognized themselves as heirs of a reflection that had begun, with
\end{abstract}

Recibido: 13/11/2018. Aceptado: 24/01/2019. 
different nuances and at different times, Nietzsche and Bataille. Several decades later, in $\mathrm{La}$ communauté désavouée, Nancy returns to the debate that had him as a protagonist in the eighties: how far did Blanchot pretend to go with the arguments that he opposed against Nancy's own text? Based on this question, which begins one of the sections of Nancy's last essay, the work intends to resume this debate in order to rethink the link between writing and community.

Keywords: community, writing, Blanchot, Nancy.

\section{Introduction}

Avec le mot grec koinonía (qui vient du mot grec koinos, qui veut dire ce qui est commun à tous, et dont la traduction à la langue française renvoie à l'idée de «communauté » ou "société ») la pensée classique, plus précisément Platon et Aristote, désignait la condition de sociabilité de l'homme'. Les grecs parlaient donc de koinonía pour décrire les formes de vie en commun à l'époque Antique. Dans la Politique, par exemple, Aristote distinguait deux formes de vie collectives, c'est-à-dire de la koinonia : celle qui avait lieu à la maison ou dans la sphère privée, qui appartient à l'oikos, et celle qui avait lieu à la cité ou dans la sphère publique, qui appartient à la polis ou à la communauté politique (ce qu'en grec on appelle la koinonía politiké) $)^{2}$. Le mot koinonía est donc le premier effort de la pensée occidentale pour aborder le problème de la communauté ${ }^{3}$.

La pensée de Hobbes, Locke et Rousseau pourraient, en ce sens, comprendre un deuxième moment. Selon les contractualistes, cependant, c'est le contrat, le pacte ou l'accord entre les hommes qui permet d'accéder à la communauté ${ }^{4}$. Mais à la différence de la pensée grecque, et en particulier à la différence de la philosophie d'Aristote, le contractualisme inverse l'ordre ontologique à partir duquel on doit concevoir le rapport entre l'homme et la communauté : c'est l'homme, autrement dit, qui occupe ontologiquement la place privilégiée dans ce rapport car ce sont les individus qui rendent possible le lien social à travers le contrat (la sociabilité de l'homme n'est

\footnotetext{
${ }^{1}$ Selon Axel Honneth, on peut traduire le terme grec koinonía par les mots latins societas et communitas. Cf. Honneth, Axel : " Comunidad. Esbozo de una historia conceptual ", dans Isegoría, Buenos Aires, numéro 20, 1999.

${ }^{2}$ Cf. Aristote : La Politique, Paris, J. Vrin, 1962.

${ }^{3}$ Cf. Alvaro, Daniel : El problema de la comunidad: Marx, Tonnies, Weber, Buenos Aires, Prometeo, 2015.

${ }^{4}$ Cf. Locke, John : Dos ensayos sobre el gobierno civil, Madrid, Espasa Calpe, 1991; Hobbes, Thomas : El leviatán, Buenos Aires, Losada, 2003 ; Rousseau, Jean-Jacques : Du contrat social, Flammarion, Paris, 2001.
} 
pas ici la condition ontologique première de l'homme, comme le montre en quelque sorte le concept le plus célèbre d'Aristote : le zoon politikon $)^{5}$.

Après la philosophie antique et la pensée contractualiste, c'est l'apparition de la Sociologie, à l'époque moderne, qui donne au problème de la communauté un nouvel élan faisant de celui-ci son objet de réflexion le plus important. La théorie sociologique, cependant, aborde ce problème d'une manière tout à fait différente à celle de la pensée classique et à celle du contractualisme. En d'autres termes, dans la tradition sociologique la notion de communauté s'oppose à la notion de société. Société et communauté ne renvoient pas à la même idée. Pour la Sociologie, la première décrit le lien social qui appartient aux sociétés modernes, tandis que la deuxième décrit le lien social qui appartient aux sociétés les plus simples, c'est-à-dire aux sociétés prémodernes ${ }^{6}$. De toute façon, le problème de la communauté demeure ici dans l'horizon théorique de la notion de la koinonía, c'est-àdire dans l'horizon délimité par la pensée grecque, car il s'agit toujours de penser la façon dont les hommes se lient à des autres hommes par le rapport que configure l'espace de la société ou de la communauté ou, pour le dire autrement, par la condition de sociabilité de l'homme.

C'est en ce sens que ce travail trouve sa pertinence : à travers le rapport entre communauté et écriture nous essaierons, autrement dit, de démontrer comment ce rapport rompt avec la notion de koinonía. C'est pourquoi nous proposerons de revenir sur l'échange qui a eu lieu entre Blanchot et Nancy dans les années 1980. Autrement : si nous voulons revenir sur ce dialogue, c'est parce que la proposition qui se déduit de cet échange permet de penser une autre communauté, c'est-à-dire une autre façon de communauté et du lien entre les hommes qui dépassent la façon dont la tradition sociologique, le contractualisme et la pensée grecque ont pensé la communauté et le lien social (ce dernier pensé toujours, bien évidemment, comme lien social). Il s'agit, en somme, de penser la temporalité et la spatialité propre du lien à partir duquel a lieu (sans avoir lieu, on y reviendra) la communauté singulière et inédite de l'écriture.

${ }^{5}$ Nous disons ici « comme le montre en quelque sorte " la notion de zoon politikon car on sait bien que cette notion décrit aussi la condition politique de l'homme chez Aristote. Le rapport entre la condition politique et la condition sociale de l'homme mérite sans doute un travail plus profond que nous ne pouvons pas développer ici. Dans la Condition de l'homme moderne, en effet, Hannah Arendt s'occupe d'analyser ceci à partir de la mauvaise traduction que, selon lui, les latins font de la Politique d'Aristote. Cf. Arendt, Hannah : Condition de l'homme moderne, Paris, Calmann-Lévy, 1983.

${ }^{6}$ Nous pouvons dire, en ce sens, que c'est la théorie sociologique allemande (Marx, Weber et Tönnies) qui commence cette opposition que l'on peut décrire très clairement à partir de l'opposition entre les mots allemands Gemeinschaft y Gesellschaft. 


\section{Communauté et écriture : le dialogue Blanchot - Nancy}

Dans le dernier essai de la série de textes concernant le sujet de la communauté, écrits par Jean-Luc Nancy et Maurice Blanchot, Nancy revient sur la phrase de Bataille qu'il avait déjà citée ailleurs et qui décrit, selon Bataille lui-même, le « sentiment de communauté » qui le liait à Nietzsche ${ }^{7}$.

C'est dans La communauté désavouée, plus précisément dans le troisième paragraphe, que Nancy revient sur cette phrase de Bataille. Mais il y revient pour essayer d'expliquer une autre phrase : celle que Blanchot a choisie pour conclure la première partie de son texte La communauté inavouable, qu'il a écrit comme une sorte de réponse au premier texte de Nancy, La communauté désœuvrée : "Ce (...) désœuvrement (...) est (...) la forme même de la " communauté désœuvrée » sur laquelle Jean Luc Nancy nous a appelés à réfléchir sans qu'il nous soit permis de nous y arrêter $»^{8}$.

Qu'il ne nous soit pas permis de nous y arrêter, ne pas s'y arrêter, cela voulait dire pour Blanchot qu'il fallait aller plus loin. Et « il faut aller plus loin » signifiait, selon Nancy, aller plus loin que ce qu'il avait proposé avec le nom de "La communauté désœuvrée ». Autrement dit, ce que la phrase de Blanchot voulait dire est donc ceci : il faut que la réflexion de Nancy se poursuive au-delà du point auquel le propre Nancy était arrivé, ne pas s’y arrêter, donc, exigeait de dépasser les limites de ce que la communauté (y compris la communauté désœuvrée) voulait dire ou pouvait vouloir dire.

Mais cette série de textes à laquelle nous renvoyons ici ne commence pas, à vrai dire, avec l'essai de Nancy sur La communauté désœuvrée (essai, en effet, paru pour la première fois en 1983 sous la forme d'un article publié dans la revue Aléa). Comme le montre l'expression citée par Nancy, elle a comme précurseur Bataille, qui avait écrit sur ce «sentiment de communauté » qui le liait à Nietzche plusieurs années avant les années 1980. À leur façon, donc, certains textes de Bataille portaient aussi sur le sujet de la communauté mais contrairement aux textes de Nancy et au texte de

${ }^{7}$ Cette série de textes relatifs au sujet de la communauté se prolonge, à dire vrai, audelà de l'échange entre Blanchot et Nancy, qui est celui sur lequel on revient ici : Giorgio Agamben publie quelques années plus tard, en 1990, La communauté qui vient. Théorie de la singularité quelconque, et Roberto Esposito, en 1998, Communitas. Origine et destin de la communauté. Cependant, notre analyse ne comprend pas ces textes car ce qui nous intéresse ici c'est le rapport entre communauté et écriture, rapport qui n'est pas présent dans les textes d'Esposito et d'Agamben. Cf. Agamben, Giorgio : La communauté qui vient. Théorie de la singularité quelconque, Paris, Seuil, 1990 et Esposito, Roberto : Communitas. Origine et destin de la communauté, Paris, PUF, 2000.

${ }^{8}$ Blanchot, Maurice : La communauté inavouable, Paris, Minuit, 1983, p. 43. 
Blanchot de façon moins systématique - ce dernier texte, soit dit en passant, fut publié la même année que l'article de Nancy et il constitue, en ce sens, le deuxième livre de la série, ou le troisième si on prend en compte les textes de Bataille - car la réflexion de Bataille sur le sujet de la communauté s'étend sur plusieurs moments de sa vie intellectuelle et toujours de façon fragmentée. On peut donc ajouter que « le sentiment de communauté » qui liait Bataille à Nietzche, selon Bataille lui-même, représente seulement un moment. Il y a d'autres moments : " la communauté de ceux qui n'ont pas de communauté » et "la communauté des amants »" sur laquelle Blanchot revient dans la deuxième partie de son texte, sont donc des formules «bataillennes » qui reflètent, en quelque sorte, ces autres moments ${ }^{10}$.

Or : la place qu'occupe cette phrase citée par Nancy pour la première fois dans La communauté désœuvrée, "le sentiment de communauté » liant Nietzsche à Bataille, venait conclure, selon lui, le dernier développement du texte dans lequel il soutenait l'existence d'une communauté qui ne serait « ni communielle, ni strictement politique ». La citation complète, cependant, disait ceci :

J'ai parlé de communauté comme existante : Nietzsche y rapporta ses affirmations mais demeura seul. (...) C'est d'un sentiment de communauté me liant à Nietzsche que nait en moi le désir de communiquer, non d'une originalité isolée ${ }^{11}$.

À la série de textes autour de la communauté, Bataille ajoute un nouveau maillon, Nietzsche : "J'ai parlé de communauté comme existante : Nietzsche y rapporta ses affirmations mais demeura seul ». Le parcours des

${ }^{9}$ Bataille aborde le thème de l'amour et de la communauté des amants dans L'Amour d'un être mortel (Paris, Rue des cascades, 2013) et dans L'Apprenti Sorcier (Paris, Éditions de la différence, 1999).

${ }^{10}$ Dans son travail « Jean-Luc Nancy y Maurice Blanchot : el reparto de lo inconfesable », Rodríguez Maciel (Escritura e imagen, Vol. 8, 2012) s'occupe de cette autre façon de penser la communauté -celle de la communauté des amants- à partir de ce dialogue entre Maurice Blanchot et Jean-Luc Nancy et l'œuvre de Margueritte Duras.

${ }^{11}$ Ce passage, on l'a déjà dit, fut cité par Nancy dans La Communauté désœuvrée (Paris, Christian Bourgois, 1999, p. 102) et écrit par Bataille dans L’Expérience intérieure. Compte tenu de l'importance qu'il a pour Nancy, pour Blanchot et pour nous-mêmes, surtout pour les pages qui suivent, je crois qu'il mérite d'être cité complètement :

"J'ai parlé de communauté comme existante : Nietzsche y rapporta ses affirmations mais demeura seul. Vis à vis de lui je brûle, comme par une tunique de Nessus, d'un sentiment d'anxieuse fidélité. Que dans la voie de l'expérience intérieure, il n'avança qu'inspiré, indécis, ne m'arrête pas : s'il est vrai que, philosophe, il eut pour fin non la connaissance mais, sans séparer les opérations, la vie, son extrême, en un mot l'expérience elle-même, Dionysos philosophos. C'est d'un sentiment de communauté me liant à Nietzsche que naît en moi le désir de communiquer, non d'une originalité isolée. ". Cf. Bataille, Georges : Euvres complètes, Paris, Gallimard, 1973, p. 39. 
auteurs et des textes sur la communauté (sur la communauté qui ne serait ni politique ni strictement communielle, on y reviendra), nous renvoie aux textes de Nancy et de Blanchot sur la communauté, et celles-ci nous renvoient en même temps aux textes de Bataille, qui nous renvoient au même temps à ceux de Nietzsche. S'il n'y a pas eu « d'originalité isolée » dans la réflexion de Bataille autour de ce sentiment de communauté, donc, c'est parce qu'elle reste toujours liée à la réflexion de Nietzsche. Et quelques décennies après cela, cette réflexion a été suivie par celles de Nancy et Blanchot dans les années 1980. Le "sentiment de communauté » dont parle Bataille ne reliait pas seulement la pensée de Bataille et celle de Nietzche, mais aussi celles de Nancy et de Blanchot, et celles de Nancy, Blanchot et Bataille lui-même. Tous ces auteurs et leurs textes, peut-on dire, ont fait partie d'une communauté de textes sur la communauté : le sentiment de communauté a été possible ici à partir du thème ou de l'objet traités. C'est le thème de la communauté, autrement dit, qui a fait communauté. Mais ce sentiment de communauté, la communauté comme objet ou thème faisant communauté, est-ce la communauté " ni communielle ni strictement politique » dont parle Nancy et puis Blanchot dans leurs réflexions (réflexions qui suivent celles de Nietzsche et de Bataille, comme Nancy et Blanchot eux-mêmes le reconnaissent) ? Il faut aller plus loin :

Je peux imaginer que cette formule de Blanchot (qu'il ne nous soit pas permis de nous y arrêter) reprend à sa façon la dernière phrase du texte (...). Elle disait " nous ne pouvons qu'aller plus loin " pour suggérer que nous avions à prolonger ce que je venais de citer de Bataille : le "sentiment de communauté me liant à Nietzsche ". Ces mots venaient conclure le dernier développement du texte : la communauté ni communielle, ni strictement politique de ceux et de cela qui se communique(nt) dans le suspens ou dans l'interruption des transmissions, des continuités d'échange - ce que je désignais sous le mot " écriture " selon un sens du mot provenant de Blanchot lui-même et de Derrida -. ${ }^{12}$

À la fin de ce passage, Nancy ajoute une note de bas de page disant ceci : " Eux-mêmes (c'est-à-dire Blanchot et Derrida) conduits vers cette valeur du mot par des frayages ouverts depuis un certain temps - on se rappelle Le Degré zéro de l'écriture de Roland Barthes, en 1953 - et dont l'histoire précise reste à faire ${ }^{13}$. À nouveau on y voit un thème ou un sujet faisant communauté. Mais il ne s'agit pas ici du thème de la communauté qui fait communauté, mais du thème de l'écriture : c'est le sujet de l'écriture, d'après ce qu'on peut déduire des phrases de Nancy, qui a fait communauté

${ }^{12}$ Nancy, Jean-Luc : La Communauté désavouée, Paris, Galilée, 2014, p. 19.

${ }^{13}$ Ibid., pp. 19-20 (note 2). 
chez Derrida, Blanchot, Barthes et Nancy lui-même. Les frayages ouverts depuis un certain temps par Roland Barthes et son texte Le Degré zéro de l'écriture, ajoute Nancy, les ont conduits vers cette valeur du mot écriture qu'il reprend en 1983 pour faire partie de cette autre communauté avec Blanchot, Derrida et Barthes.

Or, quelle est cette valeur du mot écriture ? Dans la même note de bas de page, Nancy poursuit :

Le déplacement d'un monde de l'" auteur », du "style » et de l'" œuvre " (voire du " message ») vers un espace de l' " écriture » et du " texte " (de "l'aventure d'une écriture » selon Jean Ricardou en 1967, Problèmes du Nouveau Roman, Paris, Le Seuil, p. 111), a répondu à une mutation de la perception et des conditions du sens, c'est-à-dire, de ce qui fait lien ou rapport. Le " commun " y était entièrement en jeu si l'" écriture » en venait à nommer la communication dont les pôles d'émission et de destination ne sont pas présents, sont absents à titre provisoire ou définitif. ${ }^{14}$

La valeur du mot écriture donnait ainsi un nouveau sens de ce qu'est la communauté et, pour reprendre les mots de Bataille, de ce que ce sentiment de communauté voulait dire : il s'agit de la communauté comprise comme communauté de l'écriture. Si on peut dire, d'un côté, que le thème de la communauté a fait communauté chez Nietzsche, Bataille, Blanchot et Nancy et si le sujet de l'écriture, d'un autre côté, a fait communauté chez Barthes, Blanchot, Derrida et Nancy, ce qui a fait communauté chez tous a été, autrement dit, l'écriture elle-même.

Il s'agit, selon les mots de Nancy lui-même, d'une " mutation de la perception et des conditions " de ce qui fait lien ou rapport : lien qui lie sans lier, manière d'être lié qui n'est pas pleine, c'est cela le rapport ou le lien à partir duquel a lieu la communauté de l'écriture. Sans lier là et en personne ceux qui écrivent à ceux qui sont liés par l'écriture, et sans les lier maintenant et dans le même présent, l'espace de l'écriture devient ainsi et en même temps un espace métaphysique et antimétaphysique. Espace métaphysique, en premier lieu, car ce n'est pas un espace physique, il est au-delà de la physique, de la matérialité de l'espace ordinaire dans lequel on peut se rendre présent, ou être pleinement présent, en personne. Il n'a pas, autrement dit, de spatialité et de surface, et il est donc sans limites et potentiellement infini. Ni le temps ni l'espace, ni la spatialité ni la surface ne délimitent le lien de la communauté de l'écriture.

Mais il s'agit aussi d'un espace métaphysique. Sans corps qui soient pleinement présents, sans surface pour s'appuyer, ce sont des traces qui s'y pré-

${ }^{14}$ Ibid. 
sentent, la trace de la main qui a écrit, son mouvement : trace, chose morte, et trace, chose vive, matière sans âme et animée en même temps. Si l'écriture est trace, archi-écriture, c'est en ce sens-ci, trace de la trace, geste ${ }^{15}$.

Métaphysique et antimétaphysique, donc, l'espace de l'écriture est un espace qui n'a pas de lieu : il n'a ni lieu précis, ni lieu concret. C'est ici, maintenant, qu'il a lieu, le lieu qui a lieu alors que j'écris, mais ce peut être n'importe quel lieu, le lieu où n'importe qui se lance dans le travail de l'écriture. Espace " commun », dit Nancy. Partagé. Mais partagé dans l'absence : est absent celui qui écrit tandis qu'on le lit, et est absent celui qui lit tandis que l'on écrit : "les pôles d'émission et de destination ne sont pas présents, sont absents à titre provisoire ou définitif »- rappelle Nancy -. Chaque fois qu'on lit et chaque fois qu'on écrit, chaque fois qu'un livre est ouvert pour être lu, chaque fois que l'on commence à écrire, cet espace a lieu sans que l'on puisse le toucher : espace toujours indéterminé, et auquel notre corps ne peut jamais arriver. Ni communielle ni strictement politique, la communauté à laquelle renvoie La communauté désœuvrée de Nancy, c'est donc la communauté de l'écriture.

Mais selon les mots de Blanchot, il s'agissait d'aller plus loin que là où était arrivé Nancy : il n'est pas permis - disait Blanchot - d'en rester à ce que Nancy a dit. Dans La communauté désavouée, vingt ans après cette proposition de Blanchot, Nancy reconnait qu'il n'avait pas compris du tout ce que cet "aller plus loin " de Blanchot voulait dire, même si la formule était celle que Nancy lui-même avait employée à la fin de son premier texte (et que Blanchot reprend dans La communauté inavouable, le texte qu'il écrit pour lui répondre) : "Sur le moment - écrit Nancy - je n'ai perçu cette intention que très confusément et dans l'embarras $»^{16}$.

Quelques décennies plus tard, cette perception confuse de Nancy commence à se dissiper : le nouveau sentiment de communauté que venait nommer la communauté de l'écriture ne pouvait pas être réduit à la formule que Nancy avait proposée avec le nom de la communauté désœuvrée ${ }^{17}$. Ce que Blanchot proposait, donc, était un déplacement qui devait s'ajouter à celui que Nancy avait fait à partir du mot qui décrivait la condition ni

${ }^{15}$ Pour mieux comprendre la notion de geste par rapport à l'écriture, je me permets de renvoyer à mon travail : Politique de l'écriture (Paris, L'Harmattan, 2018).

${ }^{16}$ Nancy, Jean-Luc : La Communauté désavouée, op. cit., p. 20.

${ }^{17}$ C'est en effet dans La Communauté affrontée que Nancy commence à voir se dissiper cette confusion. Paru en $2001 \mathrm{chez}$ Galilée, ce texte est le premier où il revient sur l'échange entre Blanchot et lui-même. Dans La Communauté désavouée, donc, il ne fait que développer cette révision de façon plus minutieuse. 
communielle ni strictement politique de cette mutation de la perception et des conditions de ce qui fait lien ou rapport : il faut aller plus loin, cela voulait dire, en somme, aller plus loin que le désœuvrement comme la seule condition de cette communauté. De la condition désœuvrée de la communauté, pour Blanchot, il fallait aller jusqu'à la condition inavouée de cette communauté18.

Car dans le premier essai de Nancy le désœuvrement à partir duquel on devait comprendre la condition première de cette communauté ni communielle ni strictement politique que serait la communauté de l'écriture n'était, après tout, qu'une réponse à la façon dont on a toujours compris le mot communauté, y compris le projet communiste de la communauté. Ce que Nancy voulait dénoncer, autrement dit, c'était l'idée que la communauté n'est que le processus par lequel une communauté a lieu. Cet "avoir lieu » de la communauté, ce n'est que le devenir oeuvre de la communauté : son devenir comme sujet ou substance. C'est le commun de la communauté qui devient le Peuple, la Nation ou l'État, le parti ou le prolétariat. Mais avant ce que l'on a en commun : une culture, la nation, le territoire, une idéologie ou la langue, il y a ce que " on est en commun ". Il y a un commun qui se partage, qui est partagé, mais qui n'a pas lieu, qui ne se présente pas, qui ne devient pas œuvre car il n'est pas ce qu'on a, mais ce qu'on est. C'est la présence d'un commun sans présent, qui est sans se rendre présent ${ }^{19}$.

Mais ce n'est pas seulement contre cet « avoir lieu » du commun de la communauté qu'il faut se rebeller, disait Blanchot. Car en-dessous de la condition désœuvrée de cette communauté, il y a encore quelque chose d'inavoué : en-dessous de ce lien qui n'a pas lieu, qui n'est pas plein, il y a l'inavoué de ce lien «qui fait » communauté. À la fin de son texte, Blanchot écrivait :

${ }^{18}$ Dans Maurice Blanchot. Passion politique (Paris, Galilée, 2011) Nancy ajoute que cette réflexion autour cette mutation de la perception et des conditions de ce qui fait lien ou rapport, à partir de laquelle Blanchot propose ce déplacement vers une pensée sur la condition inavouée de la communauté, commence, à dire vrai, avec les textes politiques du jeune Blanchot car la notion de la communauté inavouable qu'il développe dans les années 1980 n'est pas étrangère, selon lui, à ce qu'ont été ses convictions des années 1930. Celles-ci étaient liées, selon il y affirme, à une "interrogation fondamentale sur ce que «politique » peut vouloir dire lorsqu'il n'est plus possible d'y voir l'assomption d'une existence collective ". Ibid., p. 24. C'est moi qui souligne.

${ }^{19} \mathrm{Au}$-delà de son dialogue avec Maurice blanchot, Jean-Luc Nancy poursuit sa réflexion autour le thème de la communauté, mais depuis une autre perspective que celle qui nous intéresse ici, dans Être singulier pluriel (Paris, Galilée, 1996) et dans La comparution (Paris, Christian Bourgois Éditeur, 1997). 
La communauté inavouable : est-ce que cela veut dire qu'elle ne s'avoue pas ou bien qu'elle est telle qu'il n'est pas d'aveux qui la révèlent, puisque, chaque fois qu'on a parlé de sa manière d'être, on pressent qu'on n'a saisi d'elle que ce qui la fait exister par défaut? Alors, mieux aurait valu se taire ?20

Si le commun de cette communauté n'a pas lieu, il ne peut non plus s'avouer : on ne peut saisir de la communauté, donc, que ce qui la fait exister par défaut : le geste. La communauté de l'écriture ne se communique que par le geste car elle ne communique rien.

C'est pourquoi ce que posait Blanchot, en proposant d'aller plus loin, comprenait l'inavouable de la communauté : car ce commun que l'on est, qui se partage, il ne se communique pas. Cependant, il se transmet et il se transmet à travers l'écriture, à travers le geste qui inaugure chaque écriture $^{21}$. Et c'est cela, en effet, qui nous fait faire partie, lorsqu'on écrit, de la même communauté, c'est-à-dire de la communauté de l'écriture. Ni communielle ni strictement politique, la communauté de l'écriture appartient donc à la spatialité et à la temporalité d'une communauté tout à fait originale. Enfoncée dans l'inavoué de ce que l'on est et de ce qui y est toujours partagé, l'écriture ne communique pas car elle transmet quelque chose qui est au-delà de ce qu'elle, même ici et maintenant, à travers cette écriture qui est la mienne, communique : un mouvement, un tracé que la parole ne peut pas saisir, que le logos ne peut pas recueillir, mais que l'écriture peut toujours transmettre ou, mieux, qu'elle est la seule expérience pouvant le faire ${ }^{22}$. Il n'y a pas de parole qui puisse révéler ce que l'on est et ce qui nous lie sans lier, dans l'écriture. C'est à partir de là, en effet, que Blanchot commence aussi à questionner l'origine et le but de l'écriture. Pas l'origine (perdue) de l'écriture comprise comme le mouvement de la présence et du sens -comme le comprend Derrida- ${ }^{23}$, mais l'origine (historique) de l'écriture comprise comme l'exercice de la main qui écrit. Depuis toujours, on a expliqué cette origine en tenant compte de la raison pour laquelle on écrit : c'est pour communiquer nos pensées, nos idées, ou nos sentiments que l'écriture naît il y a plus de 35 mille ans avant notre ère ${ }^{24}$.

${ }^{20}$ Blanchot, Maurice : La Communauté inavouable, op. cit., p. 92.

${ }^{21}$ Cf. Martinez Olguín, Juan José : Politique de l'écriture, op. cit.

${ }^{22}$ Nous reprenons ce rapport entre la sphère de la communication et la sphère de la transmission, en ce qui concerne l'écriture, dans notre livre Politique de l'écriture, op. cit.

${ }^{23}$ Cf. Derrida, Jacques : De la grammatologie, Paris, Minuit, 1967.

${ }^{24}$ Cette conception qui lie l'origine de l'écriture à son but est soulignée, par exemple, par Condillac dans L'essai sur l'origine des connaissances humaines (Paris, Vrin, 2014) et, de façon plus détaillée, par Ignace Gelb (Cf. Gelb, J. Ignace : A study of writing, Chicago, University of Chicago Press, 1963). 
Mais il y a autre chose qui a à voir avec "le but » de l'écriture, et ce n'est pas lié à l'origine historique de cette pratique. Car pour Blanchot l'écriture non seulement communique, elle transmet et ce qu'elle transmet c'est le commun qu'elle seule peut transmettre et rendre présent, même si ce commun n'est pas pleinement présent car il ne se présente que par le geste, le tracé, le mouvement qui est le geste, le tracé et le mouvement de la main, de chaque main qui, en fait, ne se présente pas sur le papier. Toute écriture, peut-on donc ajouter, est toujours partagée entre ce qu'elle communique - un message, un sens - et ce qu'elle transmet - le commun qu'on est, qui est partagé - mais qui comprend aussi une singularité : une écriture unique, singulière, le geste. Et si celui-ci se transmet et ne se communique pas, c'est parce que ce qui fait lien ou rapport, ce qui fait communauté dans l'écriture, c'est-à-dire ce qui fait la communauté de l'écriture, on ne peut pas l'avouer.

\section{Mots finals : la communauté déplacée}

La communauté ni communielle ni strictement politique, ou pour reprendre les mots de Bataille, le sentiment de communauté qui le liait à Nietzsche, cette communauté (ou ce sentiment de communauté), qui trouve dans le dialogue entre Blanchot et Nancy sa formulation la plus profonde, rompt avec la tradition philosophique et sociologique, c'est-à-dire avec la tradition qu'ouvre le mot grec koinonía, dans un double sens : à travers la pensée de Nancy et la notion de la communauté désœuvrée, d'un côté, elle rompt avec l'immanentisme avec lequel cette tradition a toujours pensée la communauté. Selon cet immanentisme, très proche de la pensée sociologique et du contractualisme, la communauté ne peut se comprendre qu'à travers le processus par lequel une communauté a lieu. Celle-ci est comprise, ainsi, à partir de la façon dont le commun qui partage cette communauté devient œuvre de la communauté. Ce sont les figures du Peuple, de la Nation ou de l'État la façon dont cette pensée immanentisme de la communauté pense le lien ou le rapport entre les hommes, rapport ou lien qui devient tout de suite rapport ou lien social. En deuxième lieu, et à travers la pensée de Maurice Blanchot, cette rupture se produit à partir de la notion de la communauté inavouable (à partir de laquelle, on l'a déjà dit, Blanchot répond à Nancy). L'inavouable de la communauté s'inscrit, ainsi, dans cette ligne de rupture avec la pensée qui commence avec la réflexion grecque de la communauté car le commun qui fait communauté ne peut pas, ici, être communiqué. On peut dire, donc, que cette pensée-ci rompt avec le logocentrisme de la 
philosophie politique dans la mesure où ce n'est pas le logos ou la parole qui fait lien ou rapport (comme le dit Aristote dans les phrases célèbres de la Politique : "c'est la communauté de ces sentiments (le sentiment produit par la possession du logos, le sentiment du bien et du mal) qui engendre (...) la cité ", c'est-à-dire la koinonia politiké -ou la communauté politique-)25.

Le dialogue entre Blanchot et Nancy, on peut finalement ajouter, nous renvoie ainsi à une réflexion sur la communauté très singulière et en même temps très originale. Cette réflexion, cependant, n'amène pas seulement cet ensemble de ruptures avec la tradition occidentale de la pensée autour la communauté. Elle nous invite aussi à penser -précisément à partir de cet ensemble de ruptures- le déplacement qui se joue au sein de la notion de la communauté. Ce déplacement consiste, en un mot, à déplacer cette notion depuis la notion grecque de la communauté : celle qui décrit la pensée de la koinonia, jusqu'à la pensée de la communauté qui décrit le lien entre écriture et communauté. La communauté déplacée, titre avec lequel on a choisi de nommer ce travail, vendrait ainsi à être décrite par le mouvement que reflète cette nouvelle figure de la communauté qui désigne la communauté de l'écriture. C'est pourquoi, on peut en somme conclure, celle-ci comprend la contribution la plus profonde de cet échange intellectuel, de ce dialogue très amical, dont Maurice Blanchot et Jean-Luc Nancy ont été les animateurs. Cependant, et pour rester fidèle à la pensée de ces auteurs, cette figure de la communauté de l'écriture n'est que le premier pas sur lequel il faut avancer sans qu'il nous soit permis, pour reprendre les mots de Blanchot, de nous y arrêter. On ne peut, donc, qu'aller plus loin.

\section{Références bibliographiques}

Agamben, Giorgio : La communauté qui vient. Théorie de la singularité quelconque, Paris, Seuil, 1990.

Alvaro, Daniel : El problema de la comunidad: Marx, Tonnies, Weber,

Buenos Aires, Prometeo, 2015.

Arendt, Hannah : Condition de l’homme moderne, Paris, Calmann-Lévy, 1983.

Aristote : La Politique, Paris, J. Vrin, 1962.

${ }^{25}$ Cf. Martínez Olguín, Juan José : « Le zoon politikon, l'espace public et la proximité de la parole. Sur le logocentrisme de la philosophie politique ", dans Philosophia, numéro 78/1, 2018. 
Bataille, Georges : L'Amour d'un être mortel, Paris, Rue des cascades, 2013. Bataille, Georges: L'Apprenti Sorcier : textes correspondances et documents (1932-1939), Paris, Éditions de la différence, 1999.

Bataille, Georges : CEuvres complètes, Paris, Gallimard, 1973.

Blanchot, Maurice : La communauté inavouable, Paris, Minuit, 1983.

Condillac, Etienne Bonnot de : Essai sur l'origine des connaissances bumaines, Paris, Vrin, 2014.

Derrida, Jacques : De la grammatologie, Paris, Minuit, 1967.

Esposito, Roberto : Communitas. Origine et destin de la communauté, Paris, PUF, 2000.

Gelb, J. Ignace : A study of writing, Chicago, University of Chicago Press, 1963.

Hobbes, Thomas : El leviatán. Buenos Aires, Losada, 2003.

Honneth, Axel : "Comunidad. Esbozo de una historia conceptual ", dans Isegoría, Buenos Aires, numéro 20, 1999.

Locke, John : Dos ensayos sobre el gobierno civil, Madrid, Espasa Calpe, 1991.

Martínez Olguín, Juan José : "Le zoon politikon, l'espace public et la proximité de la parole. Sur le logocentrisme de la philosophie politique ", dans Philosophia, numéro 78/1, 2018.

Martínez Olguín, Juan José : Politique de l'écriture, Paris, L'Harmattan, 2018.

Nancy, Jean-Luc : Être singulier pluriel, Paris, Galilée, 1996.

Nancy, Jean-Luc : La comparution, Paris, Christian Bourgois Éditeur, 1997.

Nancy, Jean-Luc : La Communauté affrontée, Paris, Galilée, 2001.

Nancy, Jean-Luc : La Communauté désavouée, Paris, Galilée, 2014.

Nancy, Jean-Luc : La Communauté désœuvrée, Paris, Christian Bourgois, 1999.

Nancy, Jean-Luc : Maurice Blanchot. Passion politique, Paris, Galilée, 2011. Rodríguez Maciel, Cristina : "Jean-Luc Nancy y Maurice Blanchot: el reparto de lo inconfesable ", dans Escritura e imagen, Vol. 8, 2012.

Rousseau, Jean-Jacques : Du contrat social, Flammarion, Paris, 2001. 